\title{
PTSD Symptomatology in Neurology Nurses who Experience Verbal and Physical Abuse from Patients and Families
}

\section{Trahan $\mathbf{R}^{*}$ \\ The University of Texas Medical Branch, USA}

*Corresponding author: Roy Trahan, The University of Texas Medical Branch, 301 University Blvd. Galveston, TX 77555-1029, USA, Tel: 832-771-3863; Email: rltrahan@utmb.edu

\section{Research Article \\ Volume 2 Issue 2}

Received Date: March 28, 2018

Published Date: April 13, 2018

\section{Abstract}

Objective: The objective of this study was to assess the PTSD Impact of verbal and physical abuse on neurology nurses, identify their coping strategies, and explore relationships between demographic characteristics, incidence and coping strategies.

Background: Nurses have been identified as having the highest percentage of workplace violence and average annual rates of non-fatal violent crime greater than all occupations were found in a 2011 U.S. Department of Justice survey.

Methods: A descriptive, exploratory research design employing an online survey of 112 neurology nurses working in the United States was used for this study.

Results: Results identified the presence of PTSD as a result of verbal and physical abuse against neurology nurses, Avoidance coping strategies utilized and gender differences on multiple types of physical abuse..

Conclusion: Administrative attention must address abuse of neurology nurses and support for subsequent PTSD symptomology identified as a result of abuse by patients and families.

Keywords: Symptomatology; Hyperarousal; Nurses; Collinearity

Abbreviations: PTSD: Post-traumatic stress disorder; VPAIIS: Verbal and Physical Abuse Incidence and Intensity Scale; SPSS: Statistical Package for Social Sciences; VAPS: Violence and Aggression Scale of Patients; SPSS: Statistical Package for Social Sciences.

\section{Introduction}

The objective of this study was to assess the PTSD Impact of verbal and physical abuse on neurology nurses, identify their coping strategies, and explore relationships between demographic characteristics, incidence and coping strategies.

\section{Background and Significance}

PTSD symptomatology has been identified in health care workers at risk for abuse [1]. A growing problem has been identified for nurses in direct patient care roles, because of the risk for verbal and physical abuse [2]. A U.S. Department of Justice survey found that the average annual rate of non-fatal violent crime against nurses was 


\section{Nursing \& Healthcare International Journal}

greater than the combined average for all occupations and identified nurses as having the highest percentage of workplace violence among medical professions. Researchers have indicated that $35-80 \%$ of healthcare workers have been physically assaulted at least once, 19$80 \%$ have experienced verbal abuse, and $60-91 \%$ have experienced both verbal and physical abuse [2-6]. Patients and families have been identified as two common sources of verbal and physical abuse in the clinical environment [4,7-11].

Physical abuse is defined as "the deliberate, malicious use of physical strength for the intentional purpose of delivering harm to another individual in the form of spitting, punching, shoving, kicking, biting, scratching, stabbing or shooting the intended victim" [12]. Chapman, et al. [13] defined verbal abuse as "any communication that attacks a person professionally or personally; it may refer to behaviors such as yelling, verbal insults, or threats of harm" (p. 247). Due to the lack of preparation and overall support, coping with the violence is often difficult for nurses, potentially leading to symptoms associated with post-traumatic stress disorder (PTSD).

Pai, et al. defines PTSD as exposure to an extreme stressor or traumatic event [14]. Diagnosis of PTSD requires three groups of symptoms including re experiencing, avoidance and arousal which need to be present for a minimum of one month [14]. Hays, et al. defined coping as "the cognitive and behavioral efforts exerted to manage external and/or internal demands, which were perceived as taxing to an individual" (p. 185) [15]. Schaefer, et al. examined responses to common stressors experienced by critical care nurses and observed that aggressive coping behaviors such as accusation and sarcasm place the patient or family member in a defensive position and focus on the person rather than the problem, potentially escalating the situation. Consequently, PTSD symptomatology is a longterm risk factor and the types of coping strategies used when dealing with workplace abuse becomes an important aspect of nursing. Although incidences and nurse responses to abuse have been reported for other specialties, the high-risk neurology specialty has been largely ignored $[11,16]$.

\section{Abuse against Neurology Nurses and PTSD}

Neurology nursing is a challenging specialty dealing with complex neurological disorders and conditions that may involve aggressive patient behavior. In the qualitative meta-analysis, Management of Aggression among Demented or Brain-injured Patients, FinfgeldConnett recognized that patients with dementia or brain injury are difficult to manage due to their aggressive behaviors [17]. A study of aggressive behavior following traumatic brain injury by Baguley, et al. revealed that $25 \%$ of patients were aggressive at any given time. In addition, a study by Visscher, et al. examining the aggression of neuropsychiatric patients with acquired brain injury disclosed that $42 \%$ of patients had exhibited aggressive behavior on one or more occasions [6]. Although medical conditions may explain verbal or physical abuse by some neurology patients, Sofield, et al. suggested that verbal and physical abuse by family members toward nurses could be associated with the high stress conditions and frustration over imbalances in authority between the nurse and the family [7].

Soreny found that actual or threatened physical aggression was reported as a common occurrence in neurology clinical settings and concluded that a combination of patients' physical and psychological needs shapes the complex context in which neurology nurses deliver patient care [18]. Depending on the nurse's ability to cope over a period, the abuse often leads to PTSD symptoms that often present as the abuse continues. Some of the types of PTSD symptoms experienced by abused nurses include sleeplessness, flashbacks and depression, mild to severe anxiety and hyperarousal symptoms [14].

\section{Methods}

\section{Sample}

The sample included 112 U.S. registered nurses currently working full or part-time providing care for neurology patients. Participants were required to read and understand English, have Internet access, be employed for at least 6 months, and be at least 21 years old.

\section{Data Collection}

The study utilized three established instruments (subscales or in whole) and a bio-demographic data sheet designed specifically for the study. The first instrument was the Impact of Events Scale-Revised (IES-R) [19]. The second was the Brief COPE Inventory [20]. The third was the Verbal and Physical Abuse Incidence and Intensity Scale (VPAIIS) and a bio-demographic data sheet $[8,21,22]$.

The data collection process consisted of recruitment emails dispersed to local and regional hospitals at different intervals based on approval times at each acute care hospital over a period of three weeks beginning 


\section{Nursing \& Healthcare International Journal}

March 25, 2014, with the final hospital's recruitment emails deployed on April 10, 2014. Data collection was extended to May 25, 2014 to permit ample time for participant response in an effort to maximize participation. The acute care hospital response rate was $33.3 \%$ with 104 responses recorded.

Impact of Events Scale Revised (IES-R): Daniel Weiss and Charles Marmar developed The Impact of Events Scale - Revised in 1997 to measure the DSM-IV criteria for PTSD. The IES-R is a self-reported 22-item measure intended to subjectively assess existing distress on any number of specific life events. Of the 22 items on the IES$\mathrm{R}$, eight items measure the avoidance subscale, eight items measure the intrusion subscale, and six items measure the hyperarousal subscale. IES-R survey participants rate each item using a 5-point Likert scale of 0 (not at all) to 4 (extremely) according to the past seven days in response to a specified traumatic or stressful condition. Survey participants were asked to rate IES-R items based on their overall experience of abuse from patients and families over the past six months. Mean scores were generated for each of the three subscales. Total IES-R score were computed by summing across all items resulting in a possible range of 0 -88. A total IES-R score of 33 or higher out of 88 signifies the likely presence of PTSD symptomology. Lower scores are better [19]. For this study, a dichotomous PTSD impact variable was created for subsequent outcomes analysis using the clinical criteria of 33 or over as the split point. In addition, high and low frequency and intensity verbal and physical (patient and family) groups were created using mean and median splits on those variables.

Weiss, et al. reported that the internal consistency of the three subscales was found to be very high, with Intrusion alphas ranging from .87 to .92 , Avoidance alphas ranging from .84 to .86, and Hyperarousal alphas ranging from .79 to .90 [19]. Test-retest data for two of the samples in the study were available [19]. Sample 1 ( $\mathrm{n}=$ 429) yielded correlation co-efficients for the intrusion, avoidance, and hyperarousal subscale of .57, .51, and .59 respectively. Sample $2(\mathrm{n}=197)$ yielded correlation coefficients considerably higher at .94, .89, and .92 for intrusion, avoidance, and hyperarousal subscales. The hyperarousal subscale has proven to have good predictive validity. Content validity was available and had endorsement as high as 85\% for the intrusion and avoidance subscales, but was not available for hyperarousal.

A reliability analyses on the IES-R items showed internal consistency to be very high for the 22 items tested. The Cronbach' Alpha for the IES-R was .955. Item groupings of three subscales (Avoidance, Intrusion and Hyperarousal) were performed. Internal consistency was found to be high for eight Avoidance items tested, for eight Intrusion items tested and for six Hyperarousal items tested. The Cronbach' Alpha for the Avoidance subscale was .866, for Intrusion was .916 and for Hyperarousal was .891 .

Brief COPE Inventory: The Brief COPE Inventory measures coping stratigies used to deal with stressful or abusive events and is a condensed version of the COPE Inventory developed by Carver [23]. This research study examined three global coping scales as suggested by Carver and configured as follows: avoidance coping included self-distraction, denial, substance use, behavioral disengagement, acceptance, and self-blame; task coping included active coping, planning, humor, and positive reframing; and social/emotional coping involved use of emotional or instrumental support, venting, and religion [20]. Responses were indicated using a 4-point Likert scale (1=I usually don't do this at all to 4=I usually do this a lot) and each item was summed for each subscale, with higher scores indicating more frequent use of that coping approach. Carver previously validated the internal consistency and reliability of the Brief COPE Inventory, and reliability analyses were performed for this study found Cronbach's alphas for Avoidance coping scale, Task coping scale and Social Emotional coping scale to be .96, .905 and .929 respectively indicating internal consistency for the total scale and subscales [20].

Verbal and Physical Abuse Incidence and Intensity Scale (VPAIIS): The VPAIIS is a composite instrument compiled from three established verbal and physical abuse incidence scales [8,22,21]. The resulting 48-item questionnaire assesses abuse incidence and intensity in four categories: (a) patient verbal abuse; (b) patient physical abuse; (c) family verbal abuse; and (d) family physical abuse. The verbal abuse incidence section of the VPAIIS was adapted from the Verbally Abusive Behaviors Scale developed by Celik, et al. [8]. The physical abuse incidence section of the VPAIIS was adapted from the Violence and Aggression Scale of Patients (VAPS) developed by Lepiesova, et al. [21]. Participants rated incidence (frequency) on a 5 -point Likert scale $(0=$ never to 4=always) adapted from the 6-point Likert scale used in the VAPS [21]. 'Incidence' was defined in this case as a reflection of recalled frequency over a specified window of time and not purely new cases as used in epidemiological statistics (i.e., responses may include ongoing/continued instances). 


\section{Nursing \& Healthcare International Journal}

\section{Data Analysis}

The Statistical Package for Social Sciences (SPSS) version 22.0 was used to score, analyze, and compare the results. Results were examined for missing data, marked skewness, outliers, normality, and homogeneity. Mean/median splits on incidence provided a criterion for dichotomizing those variables into high and low sub groupings for comparative analyses.

\section{Results}

\section{Sample Demographics}

The sample was largely white (39.3\%) and Asian (34.8\%), female (84.8\%), married/living with partner (65.2\%), and had bachelor's degrees (82.1\%). The mean age was 39.96 years $(S D=10.48)$ and mean experience was 8.28 years $(\mathrm{SD}=6.82)$. No missing data, outliers, or significant heterogeneity or skewness was detected.

\section{Types of Verbal and Physical Abuse Experienced}

Table 1 displayes the descriptive analyses for the most frequent specific kinds of verbal and physical abuse encoutered from patients and families. The range of incidents reported for patient verbal and physical abuse was from 1 = never to $4=$ always. For both patients and families the majority of nurses who experienced verbal abuse from patients experienced being 'yelled or shouted at' and 'spoken inappropriately, nasty or rudely to'. More patients 'yelled or shouted' at nurses while more families 'spoke inappropriately, nasty or rudely' to the neurology nurses.

\begin{tabular}{|c|c|c|c|c|c|c|c|c|}
\hline IES-R Scales & N with score $\geq \mathbf{1}$ & \% with score $\geq \mathbf{1}$ & Score Range & \# of Items & Mean & SD & Min & Max \\
\hline Avoidance & 72 & 64.3 & 3-Jan & 8 & 0.84 & 0.753 & 0 & 4 \\
\hline Intrusion & 64 & 57.1 & 1-3 & 8 & 0.72 & 0.703 & 0 & 4 \\
\hline Hyperarousal & 47 & 42 & $4-J a n$ & 6 & 0.63 & 0.738 & 0 & 4 \\
\hline IES-R Total Score & 97 & 86.6 & Jan-66 & 22 & 16.25 & 15.09 & 0 & 88 \\
\hline
\end{tabular}

Table 1: PTSD Characteristics of Neurology Nurses Impact of Verbal and Physical Abuse from the Impact of Events Scale Revised (IES-R) $(\mathrm{N}=112)$.

For physical abuse, neurology nurses experienced the majority of physical abuse from patients as 'objects being thrown or being spit at' and 'being kicked' while the majority of physcial abuse from the families came in the form of 'thrown objects or spitting' and 'being pushed and shoved'. When comparing the types of physical abuse from patients and families we can see that both groups behaved the same by most often thowing objects or spitting at the neurology nurse. Patients' second most frequent physical abuse act was 'kicking' the nurse while families chose to 'push or shove' the nurse.

\section{PTSD Impact of Abuse and Coping on Neurology Nurses}

Table 1 shows that of the 112 participants, $86.6 \%$ had Total IES-R scores ranging from 1 - 66 which indicates the presence of at least one PTSD stress symptom after a violent event. The Avoidance Scale has the highest mean at 0.84 and the highest percentage of neurology nurse participants having a score $\geq 1$ followed by the Intrusion Scale and the Hyperarousal Scale which had $57.1 \%$ and $42 \%$ (respectively) of participants having a score of $\geq 1$. The data shows that the neurology nurse participants were more likely to use the items associated with the
Avoidance Subscale more often when dealing with the verbal and physical abuse from patients and families.

\section{Relationships between Coping and measures of PTSD Symptomatology}

Pearson correlation coefficients were computed between the subscales of all three instruments (Brief Cope Avoid, Task and Social Emote scales; Impact of Events - R, Avoidance, Intrusion, Hyperarousal and Total IESR Score; VPAIIS Mean Patient Verbal and Physical Incidence, Mean Family Verbal and Physical Incidence, Mean Patient Verbal and Physical Intensity and Mean Family Verbal and Physical Intensity). Results indicated a pattern of small significant positive relationships (higher values on one were associated with higher values on the other) between the IES subscales (indicators of PTSD symptomatology) and patient verbal and physical incidence and intensity (Table 2). A similar pattern also was evident between the IES subscales and family verbal incidence and intensity but largely lacking for family physical incidence or intensity.

For the coping subscales, only family verbal incident showed significant but small correlations with all three. 
The most robust pattern of relationships was between significant positive correlations between coping and the impact subscales which were of moderate to large magnitude indicating that higher use of all three coping strategies is related to higher scores on all three of these impact scales. Of the three, Avoidance coping shows the strongest correlations with the three impact dimensions.

Mean Family Verbal Incidence changed from a marginally significant positive correlation to slightly larger significant correlation and Mean Patient Verbal Intensity changed from a significantly positive correlation to a slightly lower marginally significant correlation of .052. The increases in strength when controlling for age and years as a neuro nurse reflect slight masking effects of age and experience whereas the decrease in strength seen with Mean Patient Verbal Intensity and Avoidance impact suggests that some of the effect was due to age and experience rather than the relationship between patient verbal abuse and avoidance impact.

\begin{tabular}{|c|c|c|c|c|c|c|}
\hline \multirow{2}{*}{$\begin{array}{l}r= \\
p< \\
n=\end{array}$} & \multicolumn{3}{|c|}{ Brief Cope Scales } & \multicolumn{3}{|c|}{ Impact of Events Scales (Ies) } \\
\hline & Avoidance & Task & Social Emote & Avoidance & Intrusion & Hyperarousal \\
\hline \multicolumn{7}{|l|}{ Verbal Incidence } \\
\hline \multirow{3}{*}{ Patient } & \multirow{3}{*}{-} & \multirow{3}{*}{-} & \multirow{3}{*}{-} & 0.244 & 0.272 & 0.3 \\
\hline & & & & 0.01 & 0.004 & 0.001 \\
\hline & & & & 112 & 112 & 112 \\
\hline \multirow{3}{*}{ Family } & 0.188 & 0.222 & 0.219 & 0.337 & 0.344 & 0.293 \\
\hline & 0.047 & 0.025 & 0.02 & 0.001 & 0.001 & 0.002 \\
\hline & 112 & 112 & 112 & 112 & 112 & 112 \\
\hline \multicolumn{7}{|c|}{ Physical Incidence } \\
\hline \multirow{3}{*}{ Patient } & \multirow{3}{*}{-} & \multirow{3}{*}{-} & \multirow{3}{*}{-} & 0.27 & 0.285 & 0.287 \\
\hline & & & & 0.004 & 0.002 & 0.002 \\
\hline & & & & 112 & 112 & 112 \\
\hline Family & - & - & - & - & - & - \\
\hline \multicolumn{7}{|l|}{ Verbal Intensity } \\
\hline \multirow{3}{*}{ Patient } & \multirow{3}{*}{-} & \multirow{3}{*}{-} & \multirow{3}{*}{-} & 0.187 & 0.319 & 0.242 \\
\hline & & & & 0.049 & 0.001 & 0.01 \\
\hline & & & & 111 & 111 & 111 \\
\hline \multirow{3}{*}{ Family } & \multirow{3}{*}{-} & \multirow{3}{*}{-} & \multirow{3}{*}{ - } & 0.278 & 0.387 & 0.325 \\
\hline & & & & 0.005 & 0.001 & 0.001 \\
\hline & & & & 102 & 102 & 102 \\
\hline \multicolumn{7}{|c|}{ Physical Intensity } \\
\hline \multirow{3}{*}{ Patient } & & 0.225 & & 0.326 & 0.379 & 0.351 \\
\hline & - & 0.031 & - & 0.002 & 0.001 & 0.001 \\
\hline & & 92 & & 92 & 92 & 92 \\
\hline & & & & & 0.567 & \\
\hline Family & - & - & - & - & 0.028 & - \\
\hline & & & & & 15 & \\
\hline IES & & & & & & \\
\hline & 0.682 & 0.629 & 0.552 & & & \\
\hline Avoidance & 0.001 & 0.001 & 0.001 & & & \\
\hline & 112 & 112 & 112 & & & \\
\hline & 0.696 & 0.594 & 0.5 & & & \\
\hline Intrusion & 0.001 & 0.001 & 0.001 & & & \\
\hline & 112 & 112 & 112 & & & \\
\hline & 0.657 & 0.572 & 0.465 & & & \\
\hline Hyperarousal & 0.001 & 0.001 & 0.001 & & & \\
\hline & 112 & 112 & 112 & & & \\
\hline & 0.725 & 0.64 & 0.543 & & & \\
\hline Total IES-R & 0.001 & 0.001 & 0 & & & \\
\hline & 112 & 112 & 112 & & & \\
\hline
\end{tabular}

Table 2: Significant Correlations between Cope X VPAIIS X IES-R Scales. 


\section{Nursing \& Healthcare International Journal}

\section{Best predictors of PTSD Symptomatology}

Due to the pattern of moderate to high correlations between some predictor variables revealed in the prior research question, collinearity statistics were run on all potential predictors then reviewed. Tolerance values exceeded .1 indicating that the assumption of nonmulticollenearity was satisfied. The higher the tolerance, the newer information a variable will contribute.
Forward stepwise regression on Total impact scores resulted in a significant model retaining three variables as significant predictors - Brief Cope Avoidance, Family Verbal Incidence and Patient Verbal Intensity - accounting for $59 \%$ of variance in Total impact. Of the variance accounted for, Brief Cope Avoidance accounted for $67 \%$. The positive relationships indicate a proportional increase in Total impact for every 1-point increase in the predictor.

\begin{tabular}{|c|c|c|c|}
\hline Variable(s) Included & $\mathbf{R}^{2}$ & Standardized $\beta$ & $\mathbf{p}<$ \\
\hline \multicolumn{4}{|c|}{ Forward } \\
\hline Model: $\mathrm{F}(3,111)=51.982, \mathrm{p}<.001$ & 0.591 & & \\
\hline BCope Avoidance & & 0.67 & 0 \\
\hline Family Verbal Incidence & & 0.224 & 0.001 \\
\hline Patient Verbal Intensity & & 0.138 & 0.027 \\
\hline \multicolumn{4}{|c|}{ Backward } \\
\hline Model: $\mathrm{F}(5,111)=34.751, \mathrm{p}<.001$ & 0.621 & & \\
\hline BCope Avoidance & & 0.569 & 0 \\
\hline BCope Task & & 0.403 & 0.005 \\
\hline BCope Social Emote & & -0.3 & 0.021 \\
\hline Patient Verbal Intensity & & 0.158 & 0.011 \\
\hline Family Verbal Incidence & & 0.238 & 0 \\
\hline
\end{tabular}

Table 3: Stepwise Regression Analyses for Total IESR - Predictors of PTSD.

Backward stepwise regression which is more inclusive and allows for the capture of synergistic effects between variables resulted in a model with five significant predictors - BCope Avoidance, BCope Task, BCope Social Emote, Patient Verbal Intensity and Family Verbal Incidence - accounting for $62 \%$ of the variance of Total impact. Brief Cope Avoidance accounted for the highest portion again, as it did in the forward regression model. Of interest is the high contribution of BCope Task which was not detected in the forward model as well as the negative relationship with BCope Social Emote reflecting a reduction in Total impact scores for every increase in Social Emote scores.

\section{Best predictors of Risk for PTSD Symptomatology}

Forward and backward stepwise logistic regressions testing the full model against a constant only model was statistically significant with BC Avoidance, Family Verbal Incidence and Family Physical Incidence reliably distinguishing between high and low risk for PTSD (Table 4). Logistic regression does not produce a true $R^{2}$ as does multiple regression but a pseudo- $\mathrm{R}^{2}$ (Nagelkerke $\mathrm{R}$ squared) can be interpreted similarly. Nagelkerke $R$ squared indicated $49 \%$ of the variance in PTSD symptomology was accounted for by the three predictors.

An additional approach to evaluating model fit is to examine the classification success of the model. The overall percentage for accurately predicting inclusion in the high PTSD symptomology group (true positives, i.e., sensitivity) was poor (41.2\%) while accurately predicting inclusion in the low PTSD symptomology group (true negatives, i.e. specificity) was excellent (98.7\%). Overall classification accuracy was good at $88.5 \%$. 


\section{Nursing \& Healthcare International Journal}

\begin{tabular}{|c|c|c|c|c|c|}
\hline \multicolumn{6}{|c|}{ Omnibus Tests of Model Coefficients } \\
\hline & Chi-square & df & \multicolumn{3}{|c|}{ Sig. } \\
\hline Model & 2.84 & 1 & \multicolumn{3}{|c|}{0.092} \\
\hline Constant & 49.284 & 12 & \multicolumn{3}{|c|}{0} \\
\hline \multicolumn{6}{|c|}{ Model Summary } \\
\hline & \multicolumn{5}{|c|}{ Nagelkerke R squared } \\
\hline Model & \multicolumn{5}{|c|}{0.49} \\
\hline \multicolumn{6}{|c|}{ Classification Table ${ }^{\mathbf{a}}$} \\
\hline \multicolumn{2}{|c|}{$\mathrm{N}$ Total IESR } & \multicolumn{4}{|c|}{ Predicted } \\
\hline \multicolumn{2}{|c|}{ Observed } & Low PTSD & High PTSD & \multicolumn{2}{|c|}{ Percent Correct } \\
\hline \multicolumn{2}{|c|}{ Low Risk PTSD } & 78 & 1 & \multicolumn{2}{|c|}{98.7} \\
\hline \multicolumn{2}{|c|}{ High Risk PTSD } & 10 & 7 & \multicolumn{2}{|c|}{41.2} \\
\hline \multicolumn{2}{|c|}{ Overall Percentage } & & & \multicolumn{2}{|c|}{88.5} \\
\hline \multicolumn{6}{|c|}{ Variables in Equation } \\
\hline & \multirow{2}{*}{ Wald } & \multirow{2}{*}{ Sig. } & \multirow{2}{*}{$\operatorname{Exp}(B)$} & \multicolumn{2}{|c|}{ 95\% C.I. for $\operatorname{Exp(B)}$} \\
\hline & & & & Lower & Upper \\
\hline BC Avoidance & 14.099 & .000 & 1.339 & 1.15 & 1.559 \\
\hline MFAVerbalINC & 5.391 & .020 & 1.276 & 1.039 & 1.567 \\
\hline MFAPhysicalINC & 3.306 & .069 & 1.807 & 0.955 & 3.42 \\
\hline Constant & 15.459 & .000 & & & \\
\hline
\end{tabular}

Table 4: Forward stepwise logistic regression of PTSD symptomology.

a. The cut value is .500

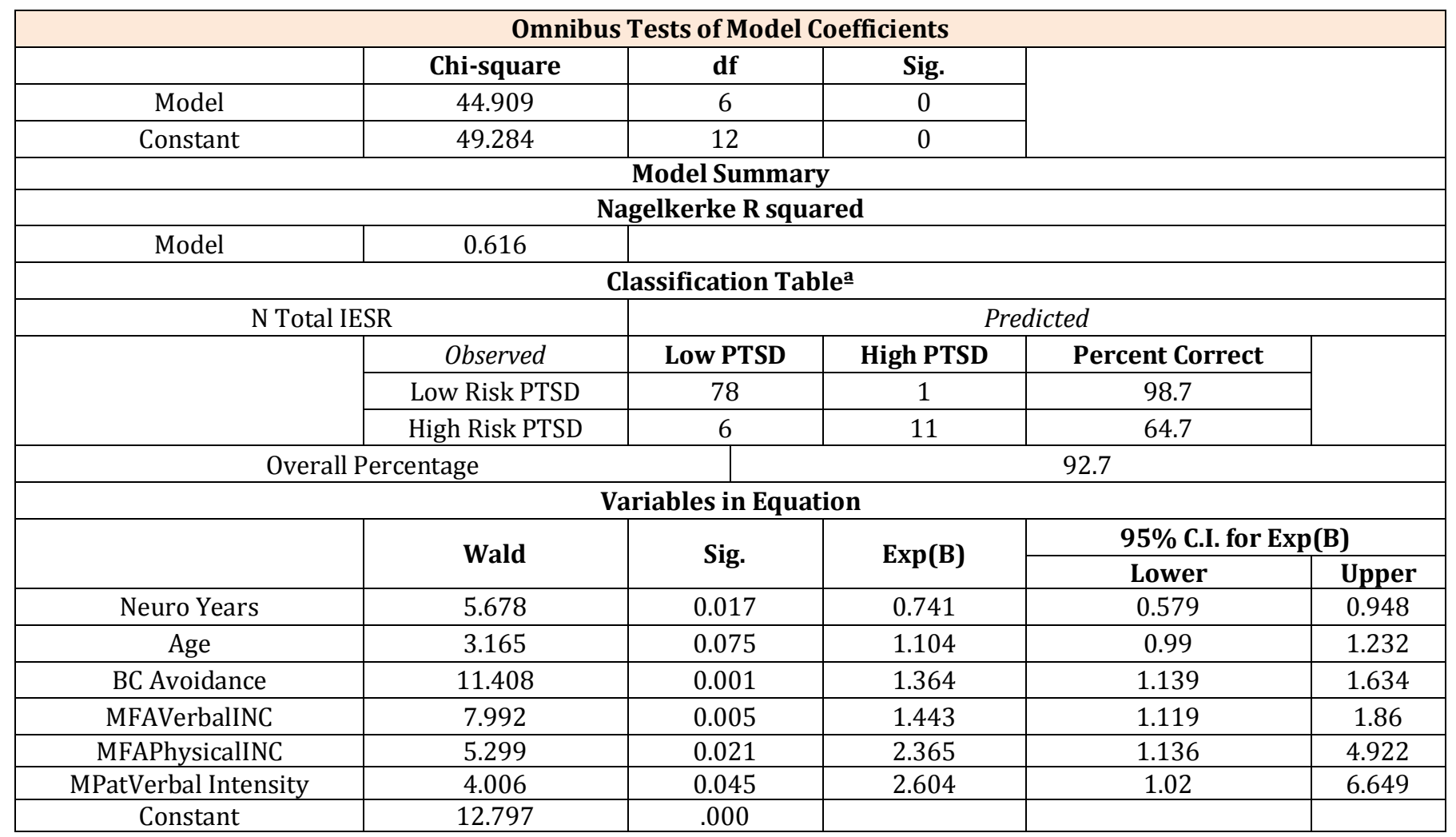

Table 5: Backward stepwise logistic regression of PTSD symptomology.

a.The cut value is .500 


\section{Nursing \& Healthcare International Journal}

The Wald statistic in the full model reveals that BC Avoidance and Mean Family Verbal Incidence are both significant, therefore it can be inferred the two variables make a significant contribution to predicting PTSD symptomology, while Mean Family Physical Incidence only contributes a marginally significant $(p=.069)$ amount to predicting risk for high PTSD symptomology. Reviewing the $\operatorname{Exp}(\mathrm{B})$ or odds ratio statistic it can be seen that BC Avoidance, Family Verbal Incidence and Family Physical Incidence are $>1$ in value, indicating that when there is an occurrence of one verbal or physical family incidence event, then the odds of belonging to the high symptomology PTSD group increases. Consequently, when a family verbal or physical abuse incidence occurs the odds are $27.6 \%$ and $80.7 \%$ respectively, that a neurology nurse will experience a high degree of PTSD symptomology. Similarly, the more a neurology nurse uses the Brief Cope Avoidance coping strategy the odds are $33.9 \%$ higher that they will exhibit higher PTSD symptomology. This implies that use of Avoidance coping may not be effective in dealing with the impact of verbal or physical abuse from patients and families.

The backward logistic regression indicated a model with six significant predictors: Neuro Years, Age, BC Avoidance, Family Verbal Incidence, Family Physical Incidence, and Mean Verbal Intensity. Model fit indices indicated a significant Hosmer and Lemeshow, indicating that the model was a poor fit compared to the constant only model. However, Nagelkerke R squared was slightly higher at .616, indicating a moderately strong relationship between the predictors and the model prediction. The overall percentage in the classification table was slightly higher than the forward LR at $92.7 \%$, with all the gain in the high-risk PTSD group, predicting true positives or sensitivity. The odds ratio for the six retained variables (Neuro Years, Age, BC Avoidance, Mean Family Verbal Incidence, Mean Family Physical Incidence, Mean Verbal Intensity) indicated five with $\operatorname{Exp}(\mathrm{B})$ scores $>1$ indicating an increase in the odds of being in the high symptomology PTSD group with an occurrence of each verbal or physical family incidence event, a unit increase in intensity, use of avoidance coping or with each additional year in age. Consequently, when a family verbal or physical abuse incidence occurs there is a $44.3 \%$ and $137 \%$, respectively, increase in the odds of a neurological nurse having high PTSD symptomatology. When a patient's verbal intensity increases 1 point, there is a $160 \%$ increase in the odds of neurology nurses having high PTSD symptomatology. Similarly, when a neurology nurse's score on the Brief Cope Avoidance coping strategy increases by one point the odds are $36.4 \%$ that they will exhibit higher PTSD symptomology. Since the Exp (b) for Age is $<1$, for each additional year in age, the odds of experiencing high PTSD symptomatology are decreased by $1 \%$.

\section{Study Limitations}

The results of this study discuss the PTSD Impact of verbal and physical abuse on neurology nurses. Findings are limited to the neurology nurse sample; therefore, results may not be generalized to the greater nursing population. Because potential participants received an email invitation to participate in the study, the inclusion/exclusion criteria may have been ignored by potential participants. Because the data collection was primarily achieved at local and regional acute care hospitals, the results may not be generalized to the national population. Because the study was conducted with English speaking participants only, findings may also be limited. The sample comprised primarily of females (84.8\%) compared to males (15.2\%). A sample comprised primarily of one gender may not have captured the experience of male neurology nurses.

\section{Discussion}

Comparison of the demograpic data with statistics for nursing demographics from the state of Texas Board of Nursing shows a much lower representation of Caucasians and a much higher representation of Asians than is indicated by the state statistics $(68.9 \%$ Caucasion, 8.1\% Asian, $88.4 \%$ female, and $37.6 \%$ have bachelors degrees) [24]. The representation of nurses with bachelor's degrees was also much lower than the state totals, but it also reflected a majority as indicated by the state statistics.

Of the 112 participants, $86.6 \%$ had Total IES-R scores ranging from $1-66$ which indicates the presence of at least one stress symptom after a violent event. The data shows that the neurology nurse participants were more likely to use the items associated with the Avoidance Subscale more often when dealing with the verbal and physical abuse from patients and families. There is a clear association between higher use of various coping strategies and higher impact, i.e., feelings of intrusion, avoidance, and hyperarousal. Coping with violent situations usually takes experience dealing with difficult patients and families. Employees, employers, patients, and families are all affected by abuse in the clinical environment.

The impact of verbal and physical abuse on neurology nurses is an important aspect of determining severity of the abuse and consequences for the nurse. Seventeen 


\section{Nursing \& Healthcare International Journal}

percent of ED nurses from one Cincinnati study scored high enough for a probable diagnosis of PTSD from patient and family related violence [1]. A substantial degree of PTSD symptomatology has been established among the neurology nurse participants of this study with $16.1 \%$ scoring greater than 33 on the Total IES-R score indicating high PTSD symptomatology. These results represent a confirmation of the current literature. Predictors of risk for high PTSD symptoms were analyzed with three variables (Avoidance coping, Family Verbal incidence and Family Physical incidence) identified as predictors of PTSD symptomatology in the forward stepwise logistic regression and six variables (years of neurological experience, age, Avoidance coping, Family Verbal incidence, Family Physical incidence and Patient Verbal intensity) identified in the backward stepwise logistic analyses as predictors of PTSD symptomatology. This becomes helpful in identification and treatment of potential victims when trying to establish relationships between coping strategies and demographic data with PTSD symptomatology.

Because of the function and purpose of coping, the type of coping employed in each situation continually varies due to reassessment of the demand and environmental variations that arise [15]. As indicated by the findings, more frequent use of certain coping strategies was indicated by the responses on the Brief COPE Avoidance subscale. Use of avoidance techniques to cope with verbal and physical abuse may be effective in the short-term, but eventually the nurse will need to address the abuse and its perpetrators in order to prevent future abuse. These findings have documented verbal and physical abuse against neurology nurses, PTSD impact of the abuse and identified common coping strategy utilization. With this new information, administrators of neurology units now have data reflecting the extent of symtomotology to which their nurses may develop PTSD as a result of daily verbal and physical abuse at the hands of patients and families. Data generated here and by subsequent research could be used by administrators to establish evidence-based practices, as well as training and support for nurses experiencing symptoms of PTSD because of verbal and physical abuse. Any changes would promote improved safety for the nurse, patient, and family members. Further research regarding PTSD as a result of verbal and physical abuse of nurses by patients and families is needed to better define the opportunities and obstacles nurses face when caring for patients and families. Future studies should focus on the nature and details of PTSD and the verbal and physical abuse against nurses, exploring other nursing specialties and examining potential causes of differences between genders. Research focused on ethnic differences in coping with verbal and physcial abuse from patient and families is also needed.

\section{References}

1. Gates DM, Gillespie GL, Succop P (2011) Violence against nurses and its impact on stress and productivity. Nurs Econ 29(2): 59-66.

2. Campbell JC, Messing JT, Kub J, Agnew J, Fitzgerald S, et al. (2011) Workplace violence: Prevalence and risk factors in the safe at work study. J Occup Environ Med 53(1): 82-89.

3. Whitehorn D, Nowland M (1997) Towards an aggression-free health care environment. The Canadian Nurse 93(3): 24-26.

4. Farrell GA, Bobrowski C, Bobrowski P (2006) Scoping workplace aggression in nursing: Findings from an Australian study. J Adv Nurs 55(6): 778-787.

5. Pich J, Hazelton M, Sundin D, Kable A (2010) Patientrelated violence against emergency department nurses. Nurs Health Sci 12(2): 268-274.

6. Visscher AJ, van Meijel B, Stolker JJ, Wiersma J, Nijman H (2011) Aggressive behaviour of inpatients with acquired brain injury. J Clin Nurs 20(23-24): 3414-3422.

7. Sofield L, Salmond SW (2003) A focus on verbal abuse and intent to leave the organization. Orthopaedic Nursing, 22(4): 274-283.

8. Celik SS, Celik Y, Agirbaş I, Ugurluoglu O (2007) Verbal and physical abuse against nurses in Turkey. Int Nurs Rev 54(4): 359-366.

9. Ahmed AS (2012) Verbal and physical abuse against Jordanian nurses in the work environment. East Mediterr Health J 18(4): 318-324.

10. Khademloo M, Moonesi FS, Gholizade H (2013) Health care violence and abuse towards nurses in hospitals in North of Iran. Glob J Health Sci 5(4): 211-216.

11. Truman A, Goldman M, Lehna C, Berger J, Topp R (2013) Verbal abuse of pediatric nurses by patients and families. Kentucky Nurse 61(1): 6-8.

12. Trahan RL, Bishop SL (2016) Coping strategies of neurology nurses experiencing abuse from patients and families. J Neurosci Nurs 48(3): 118-123. 
13. Chapman R, Styles I (2006) An epidemic of abuse and violence: Nurse on the front line. Accident and Emergency Nursing, 14(4): 245-249.

14. Pai HC, Lee S (2011) Risk factors for workplace violence in clinical registered nurses in Taiwan. Journal of Clinical Nursing 20(9-10): 1405-1412.

15. Hays MA, All AC, Mannahan C, Cuaderes E, Wallace D (2006) Reported stressors and ways of coping utilized by intensive care unit nurses. Dimensions of Critical Care Nursing 25(4): 185-193.

16. Gillespie GL, Gates DM, Berry P (2013) Stressful incidents of physical violence against emergency nurses. Online J Issues Nurs 18(1): 2.

17. Finfgeld Connett D (2009) Management of aggression among demented or brain-injured patients. Clinical Nursing Research 18(3): 272-287.

18. Soreny C (2009) Neuroscience nurses' perceptions of caring for challenging patients. British Journal of Neuroscience Nursing 5(9).
19. Weiss D, Marmar C (1997) The impact of event scale revised. In: J Wilson, et al. (Eds.), Assessing psychological trauma and PTSD. New York: Guildford.

20. Carver CS (1997) You want to measure coping but your protocol's too long: Consider the Brief COPE. International Journal of Behavioral Medicine 4(1): 92100.

21. Lepiesova M, Stork G, Ziakova C, Nemcekova M (2013) The incidence of patient aggression against nurses - psychometric properties of the measuring instrument.

22. Manderino MA, Berkey N (1997) Verbal abuse of staff nurses by physicians. J Prof Nurs 13(1): 48-55.

23. Carver CS, Scheier MF, Weintraub JK (1989) Assessing coping strategies: A theoretically based approach. J Pers Soc Psychol 56(2): 267-283.

24. Texas Board of Nursing (2014) Texas Nursing Statistics. 